\title{
Redescription disembeds relations: Evidence from relational transfer and use in problem solving
}

\author{
JAMES A. DIXON \\ University of Connecticut, Storrs, Connecticut \\ and \\ MATTHEW C. DOHN \\ Rutgers University, New Brunswick, New Jersey
}

\begin{abstract}
How relational information becomes disembedded from its original context is an important issue for theories of cognition. Two experiments tested the hypothesis that a process called redescription disembeds relations, resulting in abstract and, therefore, more transferable and robust representations. In Experiment 1, participants solved simple problems involving an alternating sequence. Participants who discovered the alternating-sequencerelation through redescription transferred the relation to a second type of problem more quickly and used it more consistently than did participants who had been directly instructed on the alternating-sequence strategy. Experiment 2 showed similar effects for participants who discovered the alternating-sequence relation through redescription, as compared with participants who had discovered the relation through information available in the display. The present results converge with previous experimental and correlational evidence that suggests that redescription creates abstract representations of relations.
\end{abstract}

An important issue for theories of cognition is how the cognitive system extracts higher order relational information from individual instances of or repeated experiences with a problem. For example, when a person learns to solve a new type of problem (e.g., the Tower of Hanoi), he or she may learn something about the relational structure that is exemplified by the problem. This relational information can then be used as a model for other problems with a similar structure but different contextual features (e.g., the missionaries and cannibals problem). Abstract relations are an essential part of many complex categories (see Ross $\&$ Warren, 2002, for a discussion) and, therefore, serve to organize much of our knowledge of the world.

A large body of work on analogical problem solving has focused on how abstract relations are extracted and represented (see Bernardo, 2001). This research has shown that both abstract and problem-specific information becomes part of the problem type schemata as a consequence of solving problems by analogy. The development of abstract representations as a result of analogical problem solving depends on the person's ability to recall a relevant previous problem and align it with the current problem (Ross \& Kilbane, 1997).

We thank Ashley Bangert, Shannon Boyer, and Christy Stum for helpful discussions regarding this project. Correspondence concerning this article should be addressed to J. A. Dixon, Department of Psychology, University of Connecticut, 406 Babbidge Road, Unit 1020, Storrs, CT 06269-1020(e-mail: james.dixon@uconn.edu).
Gentner and her colleagues (Kotovsky \& Gentner, 1996; Loewenstein \& Gentner, 2001) have proposed that abstraction can result from the act of making comparisons, rather than from consolidating or summarizing like relations across multiple instances in memory. This account, called progressive alignment, proposes that when people compare objects or problems, they align relations to maximize structural consistency. One important constraint on this process is that people prefer mappings in which elements are connected in higher order relations (i.e., systematicity). Therefore, structural alignment highlights the relational information in the problems. The highlighting, or increased salience, of relational information facilitates the extraction and re-representation of the relation. That is, the process of making a comparison causes the person to focus on relations; repeatedly focusing on relations allows the person to extract those relations from the current context. For example, Kotovsky and Gentner showed that comparing relations (e.g., symmetry or monotonic increase) within a dimension (e.g., size or saturation) facilitated comparing relations across dimensions.

The progressive alignment hypothesis has two distinct parts. First, the hypothesis posits that people structurally align relations. There is strong evidence supporting structural alignment (e.g., Gentner \& Gunn, 2001; Gentner \& Markman, 1994, 1997; Markman \& Gentner, 1993, 1996); people appear to align relations when asked to make comparisons. The second part of the hypothesis, that repeated and successive activation of a relationship facilitates the disembedding of relational information, is supported by 
more recent evidence (Loewenstein \& Gentner, 2001; Namy \& Gentner, 2002). We refer to this second part of progressive alignment as redescription.

Redescription in progressive alignment shares some important commonalities with Karmiloff-Smith's (1992) theory of representational redescription. Karmiloff-Smith proposed that representational redescription extracts relational information embedded in one's current procedures and representations. As procedures are mastered through repeated use, the embedded relational information becomes available. This relational information can then be reapplied to the problem as the basis for a new representation. For example, when children first learn to count, they can apply the appropriate labels to sets of objects but are unable to compare the magnitude of different sets by using the labels. That is, the child does not understand that the set he or she labeled "three" is smaller than the set labeled "four." However, information about magnitude is contained in the counting procedure-a four count has one more counting action than a three count. According to the representational redescription explanation, children extract the information about cardinality from the counting procedure itself (see Karmiloff-Smith, 1992, p. 104, for a discussion).

Redescription within progressive alignment is similar in that repeated focus on a relation, as a consequence of repeated comparisons, facilitates extraction of that relation. Because making a comparison is an action that emphasizes common relational structures, it may be particularly effective at offering relational information to the redescription process. Viewed this way, comparison may be highly, but not uniquely, suited to extracting relational information. Other actions that repeatedly emphasize relations should also engage redescription, so that the relational information begins to be represented abstractly.

It might be useful to contrast the account of redescription being developed here with other ways of constructing representations. Representations can, of course, be constructed through a variety of means. For example, reading a passage of text that describes the spatial layout of a scene results in a mental representation of that scene (e.g., Langston, Kramer, \& Glenberg, 1998). Similarly, research on problem solving has shown that representations of problems result from interpreting problem displays with reference to one's domain knowledge (Chi, Feltovich, \& Glaser, 1981; Wiley, 1998). Relatively small differences in how a problem is displayed can result in large differences in representation and performance. For example, Kaplan and Simon (1990) showed that a small change in how the mutilated checkerboard problem was presented strongly affected participants' representation of the problem and, subsequently, their ability to solve the problem. When parity information was presented in the display (i.e., the saturation of the individual checkerboard squares alternated), participantsincluded parity in their early hypotheses, leading to an eventual solution. These methods of constructing representations differ from redescription in two crucial respects. First, redescription involves discovering and representing information embedded in one's current representation, rather than from an external source. Second, redescription is a consequence of repeated and successive actions-actions that engage the representation in such a way that the embedded information is activated.

For example, consider the display in the upper panel of Figure 1, labeled "Balance Beam." This diagram presents three balance beams connected in a linear series by flexible joints, represented by ovals. The arrow indicates that the leftmost beam in the series will have its right arm pressed down. The question is, which way will the right arm of the last beam move? One common approach to solving this problem is to apply one's knowledge of individual balance beams and simple physical forces to construct a representation of how the movement of the first beam affects the next beam and how the movement of that beam affects the last beam. This representation contains embedded information about the alternating nature of the system; adjacent beams move in opposite directions. The alternatingsequence relation is embedded in the sense that it is observable as a consequence of solving the problem with a representation based on the simple physics of the problem, but it is not available in the problem display, nor is it the basis for the simple physics representation. Redescription extracts such embedded relationships as the relational information is exposed; repeated exposure increases the probability of redescription.

In the present study, we attempted to provide additional data on the redescription process by having some participants repeatedly engage in a problem-solving action that contained embedded information. We hypothesized that this action would result in redescription of the relation, resulting in an abstract representation of the relation. The abstract nature of the representation should affect the participants' ability to transfer and use the relation in a structurally analogous domain.

\section{EXPERIMENT 1}

In the first experiment, the participants were asked to solve a set of unfamiliar problems about a series of connected balance beams. The beams were connected by flexible joints and were shown in a linear series, as has been described above. For each problem, the participant was told that the right arm of the first beam in the series would be pushed either up or down. The participants were asked to predict the resulting movement of the final beam in the series. Half of the participants were asked to solve the problems but were not instructed on a strategy. On the basis of pilot work, we knew that most of these participants would first trace the force through the balance beam series, a strategy we call force tracing. In force tracing, participants physically simulate the movement of the beams by moving their hand in a continuous sinusoidal motion along the display. Previous work with similar physical systems (Dixon \& Bangert, 2002; Schwartz \& Black, 1996b) has strongly suggested that this strategy is based on a representation of the simple physics of the problems, as has 
B a Ia n c e B e a m
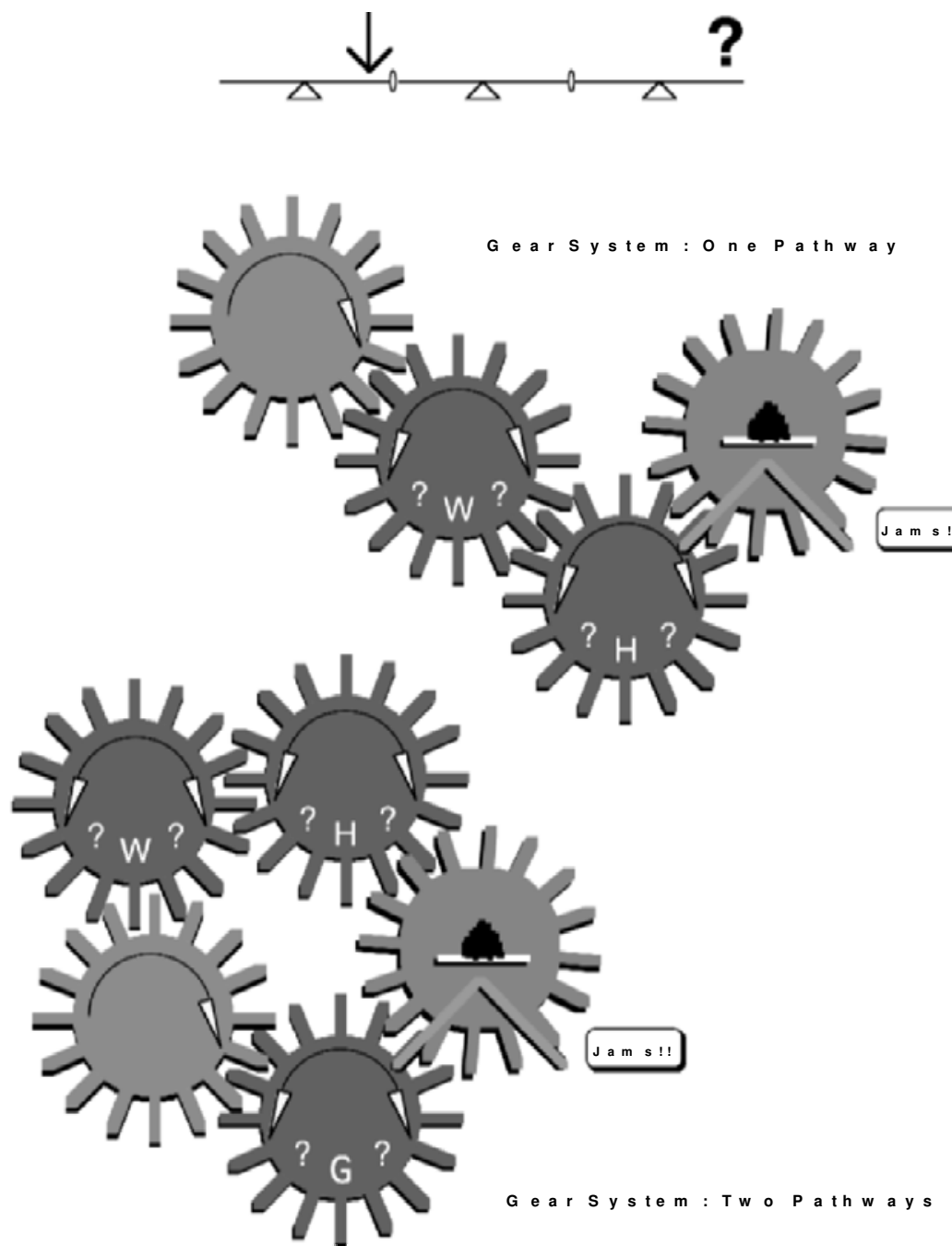

Figure 1. The top panel shows an example of a connected balance beam problem. Three balance beams are connected by flexible joints (shown as ovals). The arrow indicates that the right arm of the leftmost balance beam will be pushed down. The participants were asked to predict the motion of the right arm of the rightmost balance beam. The middle panel shows an example of a gear system problem. The gear with the single arrow (upper left) is the driving gear. The gears with the double arrows (labeled "W" and " $H$ ") are interconnecting gears. The fuel gear has a shelf that holds the fuel. When the gear turns, the fuel will fall down one of the two chutes or, if there is more than one set of interconnecting gears, the system could jam (see the text for more details). The participants were asked to select one of the two chutes or the button labeled "Jams!!" This example has a single path way connecting the driving and the fuel gears. The bottom panel shows an example of a gear system with two connecting pathways.

been described above. Most participants who use force tracing quickly discover a more efficient strategy, called updown, in which the balance beams are classified as either up beams or down beams in alternation. Past research suggests that strategies like up-down are more efficient than those that rely on simulating the physical forces of the system (Dixon \& Bangert, 2002; Schwartz \& Black, 1996b). Importantly, the force-tracing strategy contains embedded information about the up-down strategy. That is, the alternating nature of the system is available from the participants' own actions during force tracing. Therefore, we expected that the up-down strategy would be constructed through redescription for participants using force tracing; that is, they would extract the relational information from their own actions. The remaining participants were directly instructed to solve the problems using the up-down 
strategy. Thus, these participants were given instruction on a strategy that explicitly involves the alternating nature of the movement of the beams.

After solving the balance beam problems, the participants were asked to play a computer game in which they were the conductor of a train in a race. The participants were told that their train would need to obtain fuel at stations along the course. Each fueling station contained a gear system. The fuel was on a shelf attached to the last gear in the system. The first gear in the system provided the force; its movement caused the rest of the system to turn and the fuel to slide off the shelf and down one of two chutes (see the middle panel of Figure 1). To obtain fuel, the participants had to figure out which way the fuel gear would turn and position their train accordingly.

The gear systems are structurally analogous to the balance beams in that both systems are alternating sequences. The gears alternate directions, as do the balance beams. However, the two domains share few surface features, especially given the displays used here. For example, the balance beams were presented as black-and-white line drawings; the gear systems were presented on a computer screen in color. Similarly, the gear systems were presented in the context of an elaborate game; the balance beams were presented as a brief discrete task.

We predicted that the participants who discovered the up-down strategy in the balance beam task through the redescription of their own actions during force tracing would have a more abstract representation of the alternatingsequence relation and that this abstract representation would have consequences for transfer and use of the alternating-sequence relation in the gear system task. Specifically, we predicted that the participants in the redescription condition would transfer the alternatingsequence relation more quickly and easily and that use of the relation would be more stable across trials. These predictions are counterintuitivein that participants who were explicitly instructed on the up-down strategy will have used it on many more trials than those in the redescription condition and, therefore, should represent the relation more robustly.

\section{Method}

\section{Participants}

Forty-five undergraduate students (mean age $=19.21$ years, $S D=1.35$ ) participated as one option for fulfilling a course requirement. Twenty-four students participated in the redescription condition, and 21 participated in the training condition.

\section{Materials and Design}

Ten connected balance beam problems were used in the first task. Each problem showed a series of balance beams end to end in a line. Adjacent beams were connected by a flexible joint, represented by an oval. The leftmost balance beam had an arrow on the right arm of the beam. The arrow pointed either up or down. The number of balance beams in each problem ranged from three to seven. There were two problems of each size (i.e., three through seven); one problem showed the first beam with an upward-pointing arrow, and the other showed it with a downward-pointing arrow. The balance beam problems were presented as line drawings on paper. The length of each individual balance beam was 1.25 in.; the height of the fulcrum was 0.13 in.

In the second task, 32 gear system problems were presented in the context of a train race. Each gear system contained a green driving gear that provided the force to turn the other gears. An arrow on the driving gear indicated that it always turned clockwise. Each system also contained a red fuel gear. The fuel gear had a shelf on its face that held a small pile of coal. Beneath the shelf were two chutes that the fuel could slide down when the gear turned. A variable number of blue gears connected the driving and the fuel gears. Each interconnecting gear was labeled with a capital letter to facilitate the participants' referencing it and a double-headed arrow, marked by two question marks. The participants could click on an arrowhead to indicate the direction that gear would turn. Clicking on the arrowhead caused it be highlighted, the opposite arrowhead to fade, and the question marks to disappear.

To indicate which way they believed the fuel would fall, the participants clicked on the appropriate chute on the fuel gear, causing it to be highlighted. This also positioned their train so that it would catch the coal as it slid off the chute. For half of the presented gear systems, the driving and the fuel gears were connected by two pathways of gears (see the bottom panel of Figure 1 for an example). On two-pathway problems (but not on one-pathway problems), it was possible for the entire system to jam-literally fail to move because the two pathways provided opposing forces. A button labeled "Jams!!" was to the right of the chutes. The participants indicated that they believed that the system would jam by selecting this button. Selecting the Jams button positioned their train to leave the station.

The structure of the gear systems varied in a 2 (size of gear system) $\times 2$ (number of interconnecting pathways) $\times 2$ (extraneous gear) design. Small systems had four or five gears; large systems had seven or eight gears. The systems had either one or two pathways. Half of the systems contained an extraneous gear that was not relevant to the solution. Two trials presented the smaller number of gears for each level of size (i.e., four or seven), and two presented the larger number (i.e., five or eight). Half of the two-pathway problems jammed.

When the participants were satisfied with the position of the train (i.e., that it was under the appropriate chute or ready to leave a station that would jam), they clicked on the train itself. The driving and interconnecting gears, but not the fuel gear, were then hidden from view by a computer-generated screen. The fuel gear remained visible through an opening in the screen. After the screen appeared, the fuel gear either turned or jammed. Both these outcomes were accompanied by appropriate visual events (e.g., the fuel dropping) and sounds (e.g., the jammed system screeching). Feedback about the participant's judgment was first provided by (1) the visible action of the fuel gear and the resulting movement (or lack of movement of the fuel) and (2) presentation of a brief outcome message (e.g., "Got it!!" or "Missed it!!"). Feedback about the outcome was also provided in two subsequent displays. One display showed the relative speeds of the trains as they moved across the screen; a correct judgment resulted in a faster movement of the participant's train. Finally, the participants saw a small map on the right-hand side of the screen that showed the positions of their train and the opponent train.

\section{Procedure}

The balance beam problems were presented one at a time. The participants were asked to evaluate whether the right arm of the final beam in the series would be pointing "up" or "down," given the direction of movement of the first beam in the series. A three-balancebeam problem was used as an example during the initial explanation of the task. The participants in the training condition were then instructed on the up-down strategy for solving the balance beam problems as follows:

The way we'd like you to solve this problem is to classify the end of each balance beam as going either up or down. For example, in this problem 
the first balance beam is an "up beam" because its end goes up. So the second balance beam will be a "down beam", and the third one will be an "up beam". So, to solve the problem you classify each balance beam as an "up" or "down" beam.

The participants in the training condition were asked to use the updown strategy to solve all 10 problems. The participants in the redescription condition were asked simply to figure out whether the end of the last balance beam in the series would go up or down.

After completing the balance beam problems, the participants were asked to take part in a computerized train race. They were told that they would be the conductor of one train in the race; their goal was to beat the opponent train, which was controlled by the computer, to the finish line. The gear system problems were introduced as fueling stations. The participants were told that they would need fuel to complete the race; the more fuel they obtained, the faster their trains would go. A simple three-gear system was used to explain the gear system display. The participants completed four practice problems before beginning the actual trials. Two practice problems contained three gears, and two contained six gears. One system of each size had one pathway connecting the driving to the fuel gear; the other had two pathways. The participants were told to take as much time as necessary to make an accurate decision, but not longer.

The order of problems within each of the two tasks was randomized for each participant. The experiment took about $30 \mathrm{~min}$ and was run individually.

\section{Strategy Coding}

In both the balance beam and the gear system problems, the participants were instructed to "think aloud" as they solved the problems. The experimenter coded strategy use on each trial on the basis of the participants' verbalizations and actions. The following four strategies were coded in the balance beam task: force tracing, in which the movement of the beams is simulated by movement of the participant's hand; up-down, in which the beams are classified as "up" or "down" in alternation without simulating movement; skipping, in which every other beam is classified as moving the same way; and counting parity, in which the beams are counted and the parity of the count (i.e., odd or even) is used to determine whether the final beam moves the same way as the first beam.

The following five strategies were coded for the gear system task: figure eight, in which participants trace the force from the driving gear through the system (this strategy is called figure eight because the tracing action resembles a figure eight); left-right, in which the gears are classified as "left turning" and "right turning" in alternation without simulating movement (participants tend to use "left" and "right" rather than the more appropriate "clockwise" designations); skipping, in which every other gear is classified as turning the same way; and counting parity, in which the gears are counted and the parity of the count is used to determine the direction of the fuel gear. In both the balance beam and the gear system tasks, there were a few instances of strategy use that did not fit any of the categories above. We refer to these as other strategies.

Reliability was assessed by having the two experimenters independently code the videotaped sessions of 5 individuals. They had perfect agreement on $96 \%$ of the trials.

\section{Results and Discussion}

\section{Balance Beam Task}

The top part of Table 1 shows the proportions of strategy use for the participants in the redescription and the training conditions. As can be seen from the table, the participants in the training condition used the up-down strategy nearly exclusively, consistent with the instructions for that group. The participants in the redescription condition used the force-tracing strategy, as well as the up-down
Table 1

Proportion of Strategy Use Across Trials for the Balance Beam Task

\begin{tabular}{|c|c|c|c|c|c|c|c|c|c|c|}
\hline \multirow[b]{2}{*}{ Strategy } & \multicolumn{10}{|c|}{ Trial } \\
\hline & 1 & 2 & 3 & 4 & 5 & 6 & 7 & 8 & 9 & 10 \\
\hline \multicolumn{11}{|c|}{ Experiment 1: Redescription Condition } \\
\hline $\mathrm{T}$ & .83 & .75 & .75 & .71 & .58 & .50 & .50 & .50 & .50 & .50 \\
\hline ID & .17 & & .25 & .29 &.$J$ & .42 & & & 38 & .38 \\
\hline$P$ & .00 & U & .00 & .00 & .04 & .04 & .00 & 08 & .08 & .08 \\
\hline $111 \mathrm{o}$ & .00 & .00 & .00 & .00 & .04 & .04 & .04 & .04 & .04 & .04 \\
\hline \multicolumn{11}{|c|}{ Experiment 1: Training Condition } \\
\hline $\mathrm{T}$ & .00 & .00 & .00 & .00 & .00 & .00 & .00 & .00 & .00 & .00 \\
\hline JD & .95 & .95 & .95 & .95 & .95 & .95 & .95 & .95 & .95 & .95 \\
\hline P & .05 & .05 & .05 & .05 & .05 & .05 & .05 & .05 & .05 & .05 \\
\hline $111 \mathrm{c}$ & .00 & .00 & .00 & .00 & .00 & .00 & .00 & .00 & .00 & .00 \\
\hline \multicolumn{11}{|c|}{ Experiment 2: Random Condition } \\
\hline $\mathrm{T}$ & .50 & .50 & .41 & .41 & .45 & .36 & .36 & .27 & .32 & .27 \\
\hline D & .50 & .50 & .59 & .59 & .55 & .59 & .55 & .64 & .59 & .64 \\
\hline $\mathrm{CF}$ & .00 & .00 & .00 & .00 & .00 & .05 & .09 & .09 & .09 & .09 \\
\hline All & .00 & .00 & .00 & .00 & .00 & .00 & .00 & .00 & .00 & .00 \\
\hline \multicolumn{11}{|c|}{ Experiment 2: Alternating Condition } \\
\hline $\mathrm{FT}$ & .20 & .15 & .10 & .10 & .10 & .15 & .10 & .05 & .05 & .05 \\
\hline UD & .75 & .80 & .85 & .80 & .70 & .75 & .70 & .75 & .70 & .70 \\
\hline $\mathrm{CP}$ & .05 & .05 & .05 & .05 & .05 & .05 & .05 & .05 & .10 & .10 \\
\hline All others & .00 & .00 & .00 & .05 & .15 & .05 & .15 & .15 & .15 & .15 \\
\hline
\end{tabular}

Note-The proportion of strategy use for the 10 balance beam trials is shown in the cells. FT, the force-tracing strategy; UD, the up-down strategy; CP, the counting parity strategy.

strategy. As was expected, force tracing was used before the up-down strategy by most of the participants in the redescription condition; 4 participants used the up-down strategy without first using force tracing. Accuracy was very high; the only errors (2) were made by a single participant.

\section{Gear System Task}

Performance was quite accurate on the gear system task; in both conditions, the participants correctly solved $94 \%$ of the problems. A 2 (size of gear system) $\times 2$ (number of pathways) $\times 2$ (condition) analysis of variance (ANOVA) showed that the participants were significantly less accurate on problems that had two pathways $[F(1,43)=13.93$, $M S_{\mathrm{e}}=0.66$; all reported effects are significant at the .05 level]. None of the other main effects or interactions was significant. In the redescription condition, the percentages correct for the different problem types were 96\% (smallone-pathway), 90\% (small-two-pathway), 98\% (largeone-pathway), and $92 \%$ (large-two-pathway). In the training condition, the percentages correct for the different problem types were 96\% (small-one-pathway), 92\% (small-two-pathway), 96\% (large-one-pathway), and 91\% (large-two-pathway). Consistent with past research (Dixon \& Bangert, 2002), the figure eight strategy was significantly less accurate than the left-right strategy $[90 \%$ vs. $97 \% ; t(21)=2.57, S E=0.021] .{ }^{1}$

The participants in both conditions used a variety of strategies on the gear system task. The top part of Table 2 shows the proportion of strategy use across four blocks, 
Table 2

Proportion of Strategy Use by Trial Block for the Gear System Task

\begin{tabular}{llllll}
\multicolumn{5}{c}{ for the Gear System Task } \\
\cline { 2 - 6 } Strategy & 1 & 2 & $3 l o c k$ \\
\hline
\end{tabular}

Experiment 1: Redescription Condition

$\begin{array}{lllll}\text { F8 } & .60 & .39 & .35 & .30 \\ \text { LR } & .35 & .51 & .55 & .54 \\ \text { CP } & .04 & .06 & .07 & .11 \\ \text { All others } & .01 & .04 & .03 & .05\end{array}$

$\begin{array}{llll}\text { F8 } & .78 & .54 & .38 \\ \text { LR } & .20 & .35 & .36 \\ \text { CP } & .01 & .10 & .24 \\ \text { All others } & .01 & .01 & .02\end{array}$

Experiment 2: Random Condition

$\begin{array}{lllll}\text { F8 } & .60 & .45 & .40 & .36 \\ \text { LR } & .39 & .51 & .57 & .61 \\ \text { CP } & .00 & .03 & .03 & .03 \\ \text { All Others } & .01 & .01 & .00 & .01\end{array}$

\begin{tabular}{lllll} 
F8 & .68 & .42 & .41 & .29 \\
LR & .28 & .53 & .53 & .59 \\
CP & .01 & .01 & .04 & .09 \\
All others & .03 & .04 & .03 & .03 \\
\hline
\end{tabular}

Note-The proportion of strategy use for each eight-trial block is shown in the cells. F8, the figure eight strategy; LR, the left-right strategy; CP, the counting parity strategy.

with a block consisting of eight consecutive trials. Most participants first used the figure eight strategy (75\% in the redescription condition, $86 \%$ in the training condition), but use of the figure eight strategy decreased significantly across blocks $\left[F(3,129)=26.02, M S_{\mathrm{e}}=2.95\right]$. Use of the left-right and the counting parity strategies increased $\left[F(3,129)=4.55, M S_{\mathrm{e}}=4.09\right.$, and $F(3,129)=10.74$, $M S_{\mathrm{e}}=1.77$, respectively]. A 2 (condition) $\times 3$ (strategy) $\times$ 4 (block) ANOVA showed that the distribution of strategy use across blocks did not depend on condition $[F(6,258)=$ $\left.1.51, M S_{\mathrm{e}}=4.34\right]$. The skipping and other strategies were used very infrequently and, therefore, were not included in the analysis. These strategies are included in Table 2 in the row labeled "All Others."

Earlier versus later discovery. One main prediction was that the participants in the redescription condition would transfer the alternating-sequence relation to the gear system problems very quickly and that the participants in the training condition would transfer on later trials. For the gear system problems, application of the alternating-sequence relation is indicated by use of the left-right strategy. Therefore, to test this prediction, we examined the distribution of left-right discoveries (i.e., literally the first use of the left-right strategy) across trials. For example, if a participant used the left-right strategy for the first time on Trial 4, Trial 4 would be his or her discovery trial. In previous research (Dixon \& Bangert, 2002), we found that for a sample of college students $(N=$ 54 ) who completed the gear system task without any prior manipulations, the median left-right discovery trial was
Trial 8. Therefore, to assess whether the participants in the redescription condition were discovering the left-right strategy early, we compared their distribution of left-right discovery trials with this median value. The top part of Table 3 shows the frequency of left-right discovery trials relative to the median observed in previous research and the frequency of nondiscoveries in each condition. In the redescription condition, the majority of left-right discoveries occurred in the first block of seven trials (13 of 17, $76 \%$ ). However, in the training condition, significantly fewer discoveries occurred on these early trials [5 of 14 , $\left.36 \% ; \chi^{2}(1, N=31)=5.24\right]^{2}$

The early transfer of the alternating-sequence relation is consistent with the redescription hypothesis; however, an alternative explanation is that the participants in the redescription condition were reminded of solving the balance beam problems through the similarity between the figure eight and the force-tracing strategies. Recalling instances of solving the balance beam problems led to recalling use of the up-down strategy. Note that the participants in the training condition never used force tracing and, therefore, could not be reminded in this way. Under this alternative hypothesis, the difference between the training and the redescription conditions was due to the difference in whether the figure eight strategy could act as a episodic retrieval cue.

A straightforward prediction follows from this hypothesis. Because participants in the redescription condition are reminded of having used the up-down strategy in the context of the balance beam problems, application of the alternating-sequence relation to gear system problems should be affected by the superficial and structural similarities between the balance beam problems and the current gear system problem. Both superficial and structural similarity affect the mapping between base and target problems (see Ross \& Kilbane, 1997).

Recall that the gear system task contained problems with either one or two connecting pathways between the driving and the target gears. Problems with two connecting pathways are both superficially and structurally dissimilar to the balance beam problems. Superficially, the two-pathway gear problems form a closed circular pattern,

Table 3

Frequency of Earlier and Later Left-Right Discoveries in the Gear System Task

\begin{tabular}{lccc}
\hline \multicolumn{1}{c}{ Condition } & Earlier & Later & Nondiscovery \\
\hline & Experiment 1 & & \\
Redescription & 13 & 4 & 7 \\
Training & 5 & 9 & 7 \\
& Experiment 2 & \\
Random & 12 & 1 & 5 \\
Alternating & 8 & 8 & 1 \\
\hline
\end{tabular}

Note-The frequency of left-right discoveries are shown when they occurred earlier (i.e., before the median discovery trial identified in previous research) and when they occurred later (i.e., at or after the median). The frequency of nondiscoverers - that is, the participants who never used the left-right strategy-is also shown. 
rather than the open linear pattern, in the balance beam task. Structurally, the two-pathway problems require recognizing the potential for opposing forces, applying the strategy once to each pathway, and coordinating the results. Therefore, if the similarity between the figure eight strategy and force tracing remind participants of the balance beams and, ultimately, of having used the up-down strategy, the superficial and structural dissimilarities should interfere with transfer to two-pathway problems. Current models of episodic memory in problem solving predict that these effects should persist even after the initial discovery of the strategy or schema (Blessing \& Anderson, 1996; Hintzman, 1986). Consider ACT-R, in which the discovery of a new strategy is represented by the generation of a new production in declarative memory. Despite the fact that the new production is present in declarative memory, the episodic memory of previously solved examples continues to exert considerable influence until the strength of the production increases (see Blessing \& Anderson, 1996). Therefore, participants in the redescription condition should show few uses of the left-right strat- egy on two-pathway problems, as compared with participants in the training condition.

The redescription hypothesis makes a very different prediction. If redescription creates an abstract representation of the relation, participants in the redescription condition should extend the relation easily to problems that are not directly analogous to the balance beam problems. Participants in the training condition, because they do not have an abstract representation, should transfer the relation less frequently.

To test these predictions, we calculated the proportion of left-right strategy use on two-pathway systems (i.e., the frequency of left-right strategy uses on two-pathway systems, divided by the total frequency of left-right strategy uses). Consistent with the prediction from the redescription hypothesis, the participants in the redescription condition employed the left-right strategy on two-pathway systems more than did the participants in the training condition [mean proportions $=.46$ and .29 , for the redescription and the training conditions, respectively; $t(29)=$ $3.77, S E=0.045$ ]. (Only the participants who used the
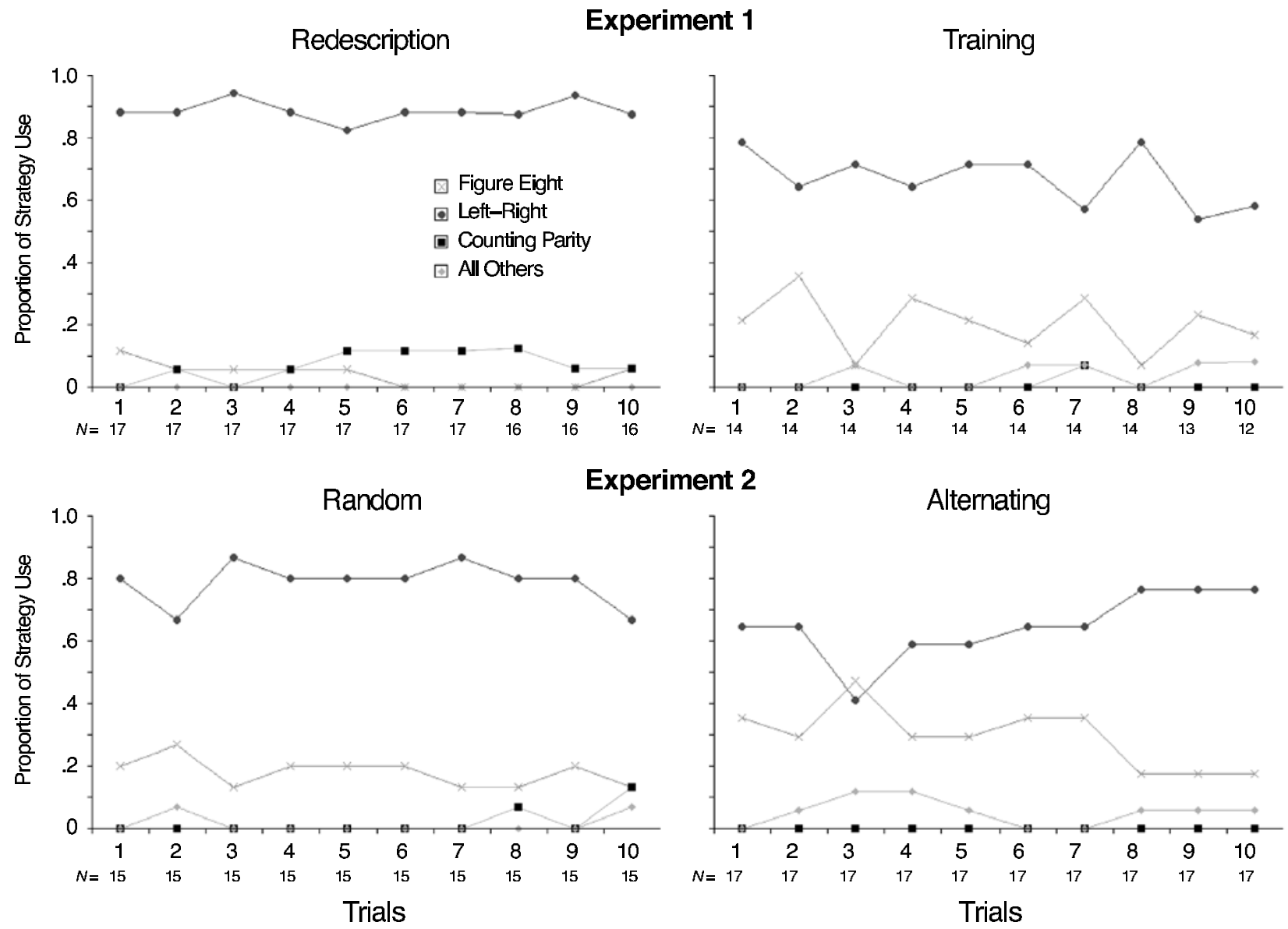

Figure 2. The proportions of strategy use on the gear system task for the 10 trials immediately following discovery of the left-right strategy is shown for Experiments 1 (top panels) and 2 (bottom panels). The number of participants contributing data on each trial is shown beneath each $x$-axis. In some conditions, the number of participants decreases slightly on the later trials because some participants discovered the left-right strategy late in the experiment and, therefore, did not have 10 trials remaining in the experiment after their discovery. 
left-right strategy are included in this analysis; the dependent variable cannot be computed if the left-right strategy was not used. For similar reasons, the remaining analyses for Experiment 1 are also conducted on the subset of the participants who used the left-right strategy.) The participants in the redescription condition showed more generalizability from the original problem context, as opposed to the interference effects that would be predicted if they were recalling a mismatching problem. ${ }^{3}$

Problem size and discovery. Consistent with a large body of work on the effects of problem structure on strategy use (e.g., Lemaire \& Reder, 1999; Siegler \& Shipley, 1995), prior research with gear systems has shown that more efficient strategies, such as left-right, are more likely to be generated when the number of gears in the system is large (Schwartz \& Black, 1996b). The figure eight strategy becomes cumbersome and more likely to produce errors when applied to larger systems. In this way, larger systems provide an impetus for generating the more efficient left-right strategy (see also Siegler \& Jenkins, 1989). Participants in the training condition, because they do not have an easily transferable representation, should be affected by problem size and, therefore, should be more likely to generate the left-right strategy on large systems. However, participants in the redescription condition should have a highly transferable representation of the alternatingsequence relation; they should transfer that relation easily to the gear system problems regardless of system size. (Recall that the number of gears in a system varied between four and eight). Consistent with this prediction, the participants in the training condition discovered the leftright strategy on larger systems (mean number of gears $=$ $6.64, S D=1.34$ ) than did the participants in the redescription condition [mean number of gears $=5.59$, $S D=1.28 ; t(29)=2.24, S E=0.469]$.

Strategy use after the discovery of the left-right strategy. Research on strategy discovery and use has also shown that after discovering a new strategy, even a highly effective strategy such as left-right, previous strategies continue to be used (e.g., Alibali, 1999; Lemaire \& Siegler, 1995). This variability reflects, in part, the tentative status of the newly discovered representation. Presumably, the representation of relational information is initially quite weak but becomes increasingly robust with repeated use. If the redescription condition creates a lasting abstract representation of the alternating-sequence relation, these fallbacks should be relatively rare. That is, because a wellformed representation is transferred, use of the new strategy will be stable. Therefore, participants in the redescription condition should fall back to the figure eight strategy less frequently than participants in the training condition. To test this prediction, we examined the distribution of strategies on the 10 trials immediately following the first use of the left-right strategy. The top panels of Figure 2 show the proportion of participants in the each condition who used each type of strategy on the 10 post-left-right discovery trials. We analyzed distribution of strategy use after the discovery of left-right strategy in a 2 (condition) $\times 10$ (post-left-right discovery trial) $\times 2$ (strategy: figure eight vs. left-right) log-linear analysis. The model containing the two-way effects provided a reasonable fit [left-right, $\left.\chi^{2}(9, N=275)=7.05, p>.63\right] .{ }^{4}$ Importantly, the condition $\times$ strategy interaction contributed significantly to the model [change in LR, $\chi^{2}(1)=22.21$ ]. The participants in the training condition were more likely to fall back to the figure eight strategy and less likely to use the left-right strategy than were the participants in the redescription condition. Consistent with the hypothesis that participants in the redescription condition transferred a stable representation to the gear system task, they reverted to the figure eight strategy infrequently, as compared with the participants in the training condition. 5

Despite the fact that the participants in the training condition used the up-down strategy on a greater percentage of balance beam trials and received instruction on the strategy, the participants in the redescription condition tended to discover the analogous strategy, left-right, more quickly in the gear system task. We also found that, as compared with the participants in the training condition, the participants in the redescription condition were more likely to apply the left-right strategy to gear system problems that were dissimilar to balance beam problems - that is, those with two pathways. This finding causes difficulty for the hypothesis that the participants in the redescription condition were reminded of the balance beam task more than were their counterparts in the training condition. Being reminded should interfere with transfer when the problems are superficially and structurally dissimilar.

Consistent with the idea that the participants in the training condition had a less transferable representation of the alternating-sequence relation, they discovered the left-right strategy on gear systems with a larger number of gears than did the participants in the redescription condition. Past research has shown that system size affects the probability of discovering more advanced strategies. Finally, the participants in the redescription condition rarely used the figure eight strategy as a fallback strategy; the participants in the training condition did so more frequently. These results are consistent with the hypothesis that redescription of the force-tracing strategy resulted in an abstract representation of the alternating-sequence relation.

Although the redescription hypothesis fits the results, an alternative explanation is that discovering the up-down strategy by whatever means would increase the likelihood of transfer, rather than the effect's being specific to the redescription process itself. That is, any process that leads to the independent discovery of a new representation, as opposed to acquiring it through some type of instruction, will create a more transferable representation. Under this hypothesis, redescription creates a more transferable representation only because it causes discovery to occur; other processes that also lead to discovery would show similar results. To address this possibility, we conducted a second experiment in which discovery of the up-down strategy was facilitated in both conditions-through re- 

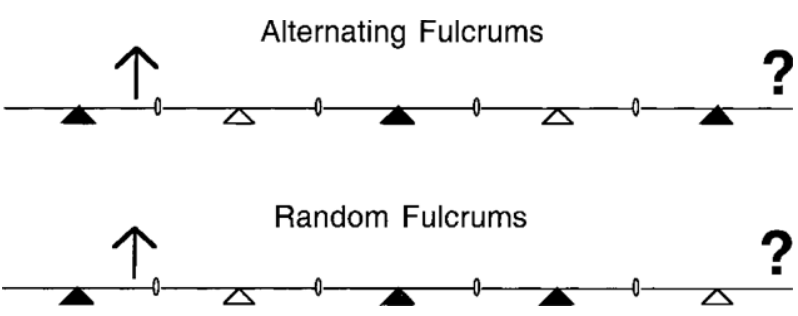

Figure 3. The top panel shows an example of a balance beam problem from the alternating-fulcrum condition. The lower panel shows an example from the random-fulcrum condition.

description in one and through a relation available in the display in the other.

\section{EXPERIMENT 2}

In Experiment 2, the participants were asked to solve the connected balance beam problems without instruction on any strategies. Half of the participants saw balance beams like those presented in the upper panel of Figure 3. These balance beams have light and dark fulcrums, and the saturation of the fulcrums alternates (i.e., dark, light, dark, etc.). The remaining participants saw balance beams like those presented in the lower panel of Figure 3. These balance beams also have light and dark fulcrums, but the saturation is not in an alternating sequence. As in Experiment 1 , the participants first solved 10 balance beam problems and then completed the gear system task.

We predicted that the participants in the alternatingfulcrum condition would be more likely to discover the alternating-sequencerepresentation of the problem through the alternating nature of the fulcrums in the display, rather than through redescribing their own actions. Kaplan and Simon (1990) showed that including parity information in a problem display can lead to the inclusion of the parity information in participants' problem representations (see also Schwartz \& Black, 1996a). The participants in the random-fulcrum condition were predicted to be more likely to discover the alternating-sequence representation through redescription; the alternating nature of the problem is less evident in the display itself. These participants will tend to discover the up-down strategy through their own mental and physical actions (i.e., force tracing), rather than through the display. Therefore, according to the redescription hypothesis, the participants in the randomfulcrum condition should be more likely than their counterparts in the alternating-fulcrum condition to construct an abstract representation of the alternating-sequence relation. Therefore, the participants in the random-fulcrum condition should show earlier transfer and more stable use of the left-right strategy, as compared with the participants in the alternating-fulcrum condition.

\section{Method}

\section{Participants}

Forty-two college students (mean age $=19.35$ years, $S D=0.98$ ) participated as one option by which to earn extra credit for a course.
Twenty-two students participated in the random-fulcrum condition, and 20 participated in the alternating-fulcrum condition.

\section{Materials and Design}

The materials were identical to those used in the first experiment, with the exception of the saturation of the fulcrums. Half of the participants saw displays in which the saturation of the fulcrums alternated. The remaining participants saw displays in which the saturation of the fulcrums changed randomly. The number of light and dark fulcrums at each level of balance beam size (i.e., three through seven) was the same across conditions. The gear system task was exactly the same as in the first experiment.

\section{Procedure}

The procedure was identical to that used in Experiment 1, except that the participants in both conditions were simply asked to solve the balance beam problems without any explicit instructions on strategies.

\section{Results and Discussion}

\section{Balance Beam Task}

The bottom part of the Table 1 shows the proportions of strategy use for the participants in each condition. Force tracing and up-down were the predominant strategies. The skipping and the counting parity strategies were used relatively rarely. As predicted, the distribution of the force-tracing and the up-down strategies was different across conditions. A 2 (condition) $\times 10$ (balance beam trial) $\times 2$ (strategy: force tracing vs. up-down) log-linear analysis compared these strategy distributions. The model containing the two-way effects fit quite well [LR, $\chi^{2}(18$, $N=382)=2.33, p>$.95]. The condition $\times$ strategy term contributed significantly to the model [change in LR, $\left.\chi^{2}(1)=40.42\right]$. When the saturation of the fulcrums alternated, the force-tracing strategy was used less, and the up-down strategy was used more.

The proportion of force-tracing strategy use was lower in the random-fulcrum condition than in the redescription condition in Experiment 1. This difference was not unexpected, because including black-and-white fulcrums helps to visually individuate the balance beams and provides some degree of alternation information. Whenever a light fulcrum is next to a dark fulcrum, there is a potential alternation cue. However, the analysis above shows that use of the forcetracing strategy was significantly greater in the randomfulcrum condition, as compared with the alternatingfulcrum condition. Therefore, the random-fulcrum condition should, as a group, have a more abstract representation of the relation, according to the redescription hypothesis.

\section{Gear System Task}

As in Experiment 1, performance was quite accurate on the gear system task. In the redescription condition, the percentages correct for the different problem types were $97 \%$ (small-one-pathway), 93\% (small-two-pathway), 97\% (large-one-pathway), and 92\% (large-two-pathway). In the training condition, the percentages correct for the different problem types were 97\% (small-one-pathway), 87\% (small-two-pathway), 99\% (large-one-pathway), and $89 \%$ (large-two-pathway). A 2 (size of gear system) $\times$ 2 (number of pathways) $\times 2$ (condition) ANOVA showed 
that the participants were significantly less accurate on problems that had two pathways $\left[F(1,40)=22.25, M S_{\mathrm{e}}=\right.$ $0.56]$. This effect depended marginally on condition $\left[F(1,40)=3.88, M S_{\mathrm{e}}=0.56, p<.06\right]$; the participants in the alternating-fulcrum condition were less accurate on twopathway problems, as compared with those in the randomfulcrum condition. It is not clear why this interaction occurred here, but not in Experiment 1. None of the other main effects or interactions was significant.

As in Experiment 1, the participants used a variety of strategies, and the distribution of strategies changed across trials. The bottom part of Table 2 shows the proportion of strategy use averaged across four blocks of eight trials each. Use of the figure eight strategy decreased across blocks $\left[F(3,120)=19.46, M S_{\mathrm{e}}=2.55\right]$; use of the left-right strategy increased significantly $[F(3,120)=$ $\left.11.31, M S_{\mathrm{e}}=3.30\right]$. The counting parity strategy was used quite infrequently and did not increase reliably across blocks $\left[F(3,120)=1.91, M S_{\mathrm{e}}=0.81\right]$. A 2 (condition) $\times$ 3 (strategy) $\times 4$ (block) multivariate ANOVA showed that the strategies used across blocks did not depend on condition $(F<1)$.

Seven participants, 3 in the alternating- and 4 in the random-fulcrum conditions, did not discover the up-down strategy. Because the purpose of this experiment was to compare the effects of discovering the alternatingsequence relation through different means, these nondiscovering participants are not included in the subsequent analyses.

Earlier versus later discovery. The bottom part of Table 3 shows the frequency of earlier and later (i.e., before and after the median discovery trial) left-right discoveries for the participants by condition. The vast majority of left-right discoveries (12 of 13, 92\%) occurred before the median for the participants in the randomfulcrum condition. The participants in the alternating condition were equally likely to discover before $(n=8)$ and after $(n=8)$ the median. Timing of left-right discoveries was significantly related to condition $\left[\chi^{2}(1, N=29)=\right.$ $6.00] .{ }^{6}$ The proportion of nondiscoveries was not significantly related to condition [ $\left.\chi^{2}(1, N=35)=2.95\right]$.

Problem size and discovery. As in Experiment 1, we examined whether the participants who discovered the leftright strategy tended to do so on larger problems. The participants in the alternating-fulcrum condition discovered the left-right strategy on larger systems (mean number of gears $=6.00, S D=2.09$ ) than did the participants in the random-fulcrum condition [mean number of gears $=$ $4.33, S D=3.05 ; t(33)=1.74, S E=0.96, p<.05$, onetailed]. As was expected, the participants who were not predicted to use redescription were more likely to discover the left-right strategy on larger systems.

Strategy use after the discovery of the left-right strategy. The bottom panels of Figure 2 show the distribution of strategies on the 10 trials immediately following the first use of the left-right strategy. A 2 (condition) $\times 10$ (post-left-right discovery trial) $\times 2$ (strategy: figure eight vs. left-right) log-linear analysis compared these strategy distributions. The model containing the two-way effects fit quite well [LR, $\left.\chi^{2}(9, N=281)=4.82, p>.80\right]$. The condition $\times$ strategy term contributed significantly to the model [change in LR, $\chi^{2}(1)=4.12$ ]. The participants in the random-fulcrum condition were less likely to fall back to the figure eight strategy after discovering the left-right strategy than their counterparts in the alternating-fulcrum condition.

In the second experiment, the participants in the randomfulcrum condition were more likely to discover the leftright strategy early in the gear system task, as compared with the participants in the alternating-fulcrum condition. The participants in the random-fulcrum condition also discovered the left-right strategy on smaller gear systems. Following discovery, their use of the left-right strategy was more stable than that of the participants in the alternating-fulcrum condition; they used the figure eight strategy as a fallback strategy infrequently. In summary, the transfer and use of the alternating-sequence relation by the participants who were predicted to discover through redescription is consistent with the hypothesis that they formed a more abstract representation of the relation than did the participants who had the relation easily available in the display. Therefore, the second experiment suggests that redescription, rather than discovery of the relation per se, was the important factor in creating an abstract relation.

\section{GENERAL DISCUSSION}

One might reasonably have expected that using the updown strategy on more balance beam trials would lead to a stronger representation and greater activation of the alternating-sequence relation and, therefore, to an increase in the likelihood of transfer and use. However, both experiments showed that discovery through redescription facilitated transfer and use more than did repeated applications in a previous problem domain. The participants who were instructed on the up-down strategy (Experiment 1) and those who discovered it through the display (Experiment 2) used the up-down strategy on more balance beam trials than did the participants in the redescription and the random-fulcrum conditions. However, both of these groups tended to discover the left-right strategy later in the experiment, their discoveries appeared to be more strongly affected by task factors, and they were more likely to fall back to the previously successful figure eight strategy.

The two experiments reported here jointly suggest that redescription disembeds relations, resulting in an abstract representation of the relation. This representation appears to be more easily transferred to a new problem domain and to be used more consistently after its initial discovery. Redescription may be one means by which people extract relational information from individual or successive instances. It may, therefore, be important for our understanding of how categories that include relational information are formed. 
Redescription may also provide a way for the cognitive system to focus attention on relations that are likely to be particularly relevant for problem solving. Given the myriad relations that are available in even a slightly complex problem, it is necessary to explain why particular relations and not others are selected. For example, consider the spatial layout of the gear system shown in the middle panel of Figure 1. There is almost no limit to the number of relations a naive problem solver could propose on the basis of the spatial characteristics of the display (e.g., height of the driving gear relative to the fuel gear or angle of the connecting gear to the fuel gear). Therefore, it is necessary to explain how a problem solver's attention becomes focused on the relevant relations. Part of the explanation may be that redescription narrows the field to include only those relations that are part of one's current solution.

In addition to the left-right strategy, the participants discovered the counting parity and the skipping strategies. The counting parity strategy is of particular interest because it represents the discovery of a mathematical relation during problem solving. Dixon and Bangert (2003) have presented evidence consistent with the hypothesis that the counting parity strategy is discovered through a process like progressive alignment. The probability of discovering the counting parity strategy increased as participants solved problems that required comparing two pathways with the same parity but with a different number of gears. Because the skipping strategy was rarely discovered, testing predictions about its origins would be difficult.

The results of the present study are consistent with the account of redescription offered as part of the progressive alignment hypothesis (Kotovsky \& Gentner, 1996). Repeated successive activation of a relation leads to the extraction of the relation from its original context. In progressive alignment, this activation occurs because relations are highlighted each time a comparison between two objects or situations is made. Comparison requires structural alignment of relations and is, therefore, an effective means for strongly activating relational information. In the present study, activation of the relevant relation, alternating sequences, was predicted to occur each time participants solved a balance beam problem by using a representation of the simple physics of the system (i.e., how movement of the first balance beam would affect the subsequent beam and how movement of that beam would affect the next beam, etc.). The alternating nature of the system is embedded in this representation of the problem.

An alternative explanation, also consistent with the progressive alignment account, is that the redescription (Experiment 1) and random-fulcrum (Experiment 2) conditions facilitated the comparison of successive problems and, therefore, these conditions were more effective at engaging progressive alignment. However, this explanation quickly runs into difficulty. The graphic displays in Experiment 1 were identical across conditions and, therefore, cannot have been the source of a difference in facilitating comparison. Perhaps one might argue that being instructed on the up-down strategy somehow inhibited the compar- ison of successive problems. But this argument will not work for Experiment 2, in which the instructions were identical. The only difference between the conditions in Experiment 2 was in the graphic displays. However, the displays in the alternating-fulcrum condition should be more easily alignable than the displays in the random-fulcrum condition, because the former displays have better structure. Therefore, the alternating-fulcrum condition should show better transfer and more stable use than the randomfulcrum condition.

Therefore, we argue that the present results provide an alternative and converging type of evidence for the redescription aspect of the progressive alignment hypothesis. It appears that repeatedly solving even a fairly simple problem can lead to redescription, if the solution strategy contains an embedded relation.

Recent work by Dixon and Bangert (2002) supports the possibility that redescription can occur as a consequence of repeated actions during problem solving. Dixon and Bangert (2002) examined how a participant's history of performance on a problem-solving task predicted the discovery of a new strategy on subsequent trials. They showed that the probability of discovering a new strategy was jointly affected by (1) having a history of accuracy with a lower strategy that contained the relevant embedded information and (2) using that strategy in repeated succession just prior to the discovery trial. They suggested that relations are extracted and stored as an aspect of the problem representation during repeated accurate use. Concentrated use of the strategy prior to the discovery trial strongly activates the relation, increasing its salience and the likelihood of applying the relation as part of a problem-solving strategy. The present study complements these previous correlational results by showing that repeated problem solving may also produce the type of abstract relational representations predicted by redescription.

\section{REFERENCES}

Alibali, M. W. (1999). How children change their minds: Strategy change can be gradual or abrupt. Developmental Psychology, 35, 127-145.

Bernardo, A. B. I. (2001). Principle explanation and strategic schema abstraction in problem solving. Memory \& Cognition, 29, 627-633.

Blessing, S. B., \& Anderson, J. R. (1996). How people learn to skip steps. Journal of Experimental Psychology: Learning, Memory, \& Cognition, 22, 576-598.

Chi, M. T. H., Feltovich, P. J., \& Glaser, R. (1981). Categorization and representation of physics problems by experts and novices. Cognitive Science, 5, 121-152.

Dixon, J. A., \& BAngert, A. S. (2002). The pre-history of discovery: Precursors of representational change in solving gear-system problems. Developmental Psychology, 38, 918-933.

Dixon, J. A., \& BAngerT, A. S. (2003). On the spontaneous discovery of a mathematical relation during problem solving. Manuscript submitted for publication.

Gentner, D., \& GunN, V. (2001). Structural alignment facilitates the noticing of differences. Memory \& Cognition, 29, 565-577.

Gentner, D., \& Markman, A. B. (1994). Structure alignment in comparison: No difference without similarity. Psychological Science, 5, 152-158.

Gentner, D., \& Markman, A. B. (1997). Structure mapping in analogy and similarity. American Psychologist, 52, 45-56. 
Hintzman, D. L. (1986). "Schema abstraction" in a multiple-trace memory model. Psychological Review, 93, 411-428.

Kaplan, C. A., \& Simon, H. A. (1990). In search of insight. Cognitive Psychology, 22, 374-419.

Karmiloff-Smith, A. (1992). Beyond modularity: A developmental perspective on cognitive science. Cambridge, MA: MIT Press.

Kotovsky, L., \& Gentner, D. (1996). Comparison and categorization in the development of relational similarity. Child Development, 67, 2797-2822.

Langston, W., Kramer, D. C., \& Glenberg, A. M. (1998). The representation of space in mental models derived from text. Memory \& Cognition, 26, 247-262.

Lemaire, P., \& Reder, L. (1999). What affects strategy selection in arithmetic? The example of parity and five effects on product verification. Memory \& Cognition, 27, 364-382.

Lemaire, P., \& Siegler, R. S. (1995). Four aspects of strategic change: Contributions to children's learning of multiplication. Journal of Experimental Psychology: General, 124, 83-97.

LoEWENSTEIN, J., \& GENTNER, D. (2001). Spatial mapping in preschoolers: Close comparisons facilitate far mappings. Journal of Cognition \& Development, 2, 189-219.

Markman, A. B., \& Gentner, D. (1993). Structural alignment during similarity comparisons. Cognitive Psychology, 25, 431-467.

Markman, A. B., \& Gentner, D. (1996). Commonalities and differences in similarity comparisons. Memory \& Cognition, 24, 235-249.

NAmy, L. L., \& GenTner, D. (2002). Making a silk purse out of two sow's ears: Young children's use of comparison in category learning. Journal of Experimental Psychology: General, 131, 5-15.

Ross, B. H., \& Kilbane, M. C. (1997). Effects of principle explanation and superficial similarity on analogical mapping in problem solving. Journal of Experimental Psychology: Learning, Memory, \& Cognition, 23, 427-440.

Ross, B. H., \& WARREN, J. L. (2002). Learning abstract relations from using categories. Memory \& Cognition, 30, 657-665.

Schwartz, D. L., \& Black, J. B. (1996a). Analog imagery in mental model reasoning: Depictive models. Cognitive Psychology, 30, 154-219.

Schwartz, D. L., \& BLACK, J. B. (1996b). Shuttling between depictive models and abstract rules: Induction and fallback. Cognitive Science, 20, 457-497.

SIEGLER, R. S., \& JENKINS, E. (1989). How children discover new strategies. Hillsdale, NJ: Erlbaum.

Siegler, R. S., \& ShIPley, C. (1995). Variation, selection, and cognitive change. In T. Simon \& G. Halford (Eds.), Developing cognitive competence: New approaches to process modeling (pp. 31-76). Hillsdale, NJ: Erlbaum.

WiCKENS, T. D. (1989). Multiway contingency tables for the social sciences. Hillsdale, NJ: Erlbaum.

WiLEY, J. (1998). Expertise as mental set: The effects of domain knowledge in creative problem solving. Memory \& Cognition, 26, 716-730.

\section{NOTES}

1. This analysis was conducted on the subsample of participants who used both the figure eight and the left-right strategies $(n=22)$. However, the larger samples of participants who ever used the figure eight strategy $(n=36)$ and who ever used the left-right strategy $(n=31)$ had the same proportions correct for the respective strategies as this smaller subsample of joint users. All comparisons of mean proportions reported in the manuscript were done on both raw scores and arcsine transformed scores. In all cases, these analyses showed very strong agreement; no result changed from significant to nonsignificant or vice versa. The raw score analyses are reported.

2 . There were 7 nondiscoverers in each condition. The relationship between condition and discovering the left-right strategy still holds if nondiscoverers are included in the analysis as right-censored cases (i.e., assuming that nondiscoverers would have eventually discovered). When nondiscoverers are included in the late-discovering category (i.e., post-Trial 7), the relationship between condition and discovering the left-right strategy remains significant $\left[\chi^{2}(1, N=45)=4.30\right]$. Nor does the pattern change appreciably if nondiscoverers are considered as a separate category $\left[\chi^{2}(2, N=45)=5.30, p<.05\right.$, one-tailed].

3 . An alternative formulation of the reminding hypothesis is that the effects of surface and structural similarity will be limited to the discovery of the left-right strategy. Participants in the redescription condition should discover left-right less frequently on two-pathway problem. Nine participants discovered the left-right strategy on two-pathway problems4 in the redescription condition and 5 in the training condition. Contrary to the reminding hypothesis, whether the participants discovered on oneor two-pathway problems was not related to condition $\left[\chi^{2}(1, N=31)=\right.$ $0.55]$.

4. In this time series analysis, $N$ is the total number of figure eight and left-right strategy uses (see Wickens, 1989, pp. 28-29, for a discussion of log-linear analysis of time series data).

5. An alternative approach to testing this prediction is to compute the proportion of figure eight strategy use immediately following each use of the left-right strategy (i.e., on the subsequent trial). For example, if a participant used the left-right strategy on Trials 3, 4, and 6 and the figure eight strategy on Trials 5 and 7, he or she would have two fallbacks out of three left-right uses (proportion of fallbacks $=.67$ ). Consistent with the log-linear analysis reported in the text, the participants in the redescription condition rarely used the figure eight strategy after the leftright strategy (mean proportion of fallbacks $=.02, S D=.06$ ); the participants in the training condition did so much more frequently [mean proportion of fallbacks $\left.=.21, S D=.30 ; F(1,29)=6.19, M S_{\mathrm{e}}=0.043\right]$. Interestingly, the effect of condition did not depend on whether the problem that occurred subsequent to using the left-right strategy contained one or two pathways $\left[F(1,29)=2.56, M S_{\mathrm{e}}=0.03, p<.12\right.$; redescription, mean proportion of fallbacks $=.02$ and .03 ; training, mean proportion of fallbacks $=.14$ and .28 , for one- and two-pathway problems, respectively]. This suggests that falling back to the figure eight strategy is not due entirely to difficulty with the two-pathway problems.

6 . This relationship remains significant if participants who did not discover the left-right strategy are included as a separate category $\left[\chi^{2}(2\right.$, $N=35)=8.89$ ].

(Manuscript received May 27, 2002, revision accepted for publication June 5, 2003.) 